\title{
Low back pain in a patient with malignancy (2010: 4a)
}

(C) European Society of Radiology 2009

\section{Case report}

A 16-year-old boy with a right sacral Ewing sarcoma underwent an almost complete resection, leaving only a small intramedullary component in place. Immediately following surgery, he received adjuvant chemotherapy according to the EURO-EWING 99 localised disease protocol. Radiotherapy was initiated 1 month after finishing the VIDE protocol. A total dose of $64 \mathrm{~Gy}(40 \times$ $1.6 \mathrm{~Gy})$ was given. Oedema in the skin, subcutaneous tissues and paravertebral muscles was evident on the MR examination immediately following surgery and during irradiation, which subsided upon termination of the treatment. Consolidation chemotherapy was started after the first two radiotherapy sessions and was completed 6 months later.

The patient started complaining of gradually increasing low back pain and skin sensitivity 6 months after the end of the radiotherapy. Upon clinical examination erythema of the skin and a painful palpation were evident. An MR study was performed (Fig. 1).

What is the diagnosis?

Readers are invited to supply one possible diagnosis via electronic means to: robert.hermans@uzleuven.be

The subject of the email should include 'Interpretation Corner' and the number given above (e.g. Interpretation Corner 2010: 4a).

They should include their name, title, address, FAX and phone number. date.

Deadline: one clear calendar month from distribution

The names of the first 25 radiologists submitting the correct diagnosis will be published (only one from any individual centre and none from the host institution!).

Three months after the initial publication of the case history, the authors will publish the final diagnosis
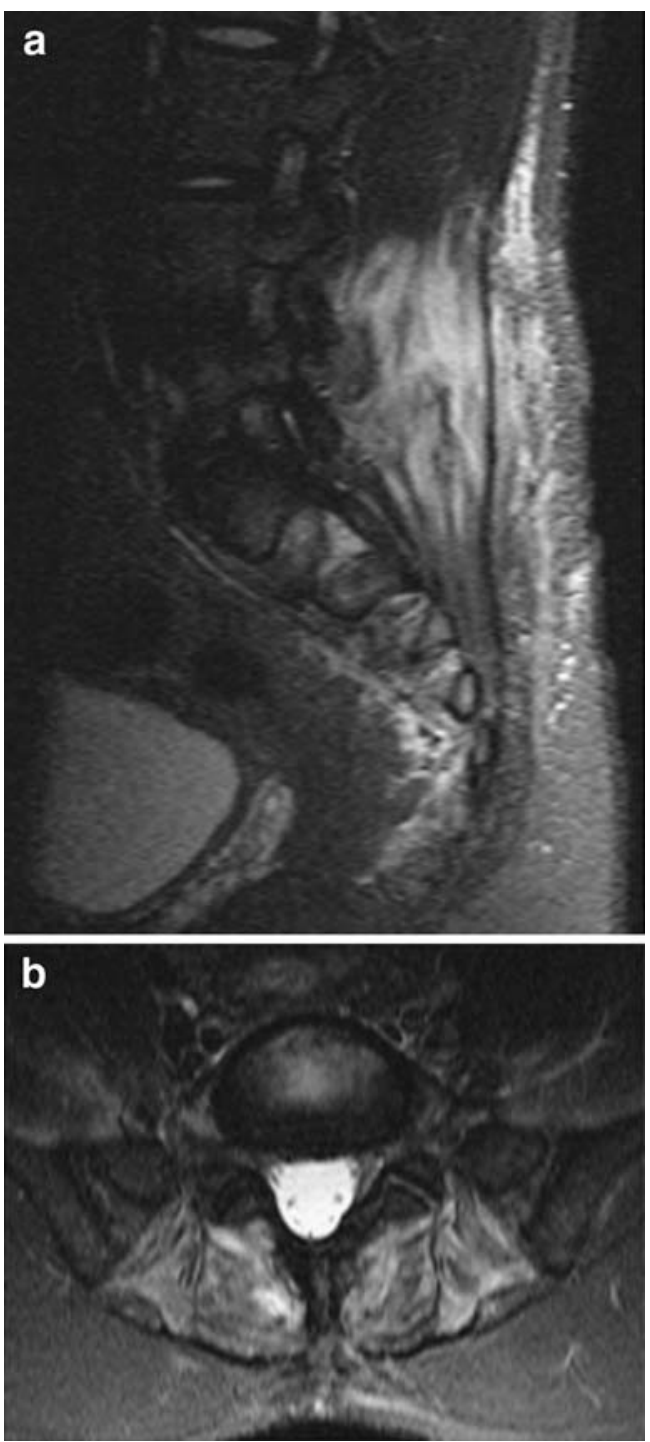

Fig. 1 Sagittal (a) and axial (b) IR image, 6 months after radiotherapy and 7 months after the start of chemotherapy 